\title{
Shocks in the asymmetric exclusion process with internal degree of freedom
}

\author{
Fatemeh Tabatabaei ${ }^{1, *}$ and Gunter M. Schütz ${ }^{1,2, \uparrow}$ \\ ${ }^{1}$ Institut für Festkörperforschung, Forschungszentrum Jülich, 52425 Jülich, Germany \\ ${ }^{2}$ Interdisziplinäres Zentrum für komplexe Systeme, University of Bonn, Germany
}

(Received 8 August 2006; published 10 November 2006)

\begin{abstract}
We determine all families of Markovian three-state lattice gases with pair interaction and a single local conservation law. One such family of models is an asymmetric exclusion process where particles exist in two different nonconserved states. We derive conditions on the transition rates between the two states such that the shock has a particularly simple structure with minimal intrinsic shock width and random walk dynamics. We calculate the drift velocity and diffusion coefficient of the shock.
\end{abstract}

DOI: 10.1103/PhysRevE.74.051108

PACS number(s): 05.70.Ln, 82.40.Fp, 02.50.Ga

\section{INTRODUCTION}

The asymmetric simple exclusion process (ASEP) $[1,2]$ has often been called the Ising model of nonequilibrium statistical physics. In this stochastic lattice gas model, particles move randomly with a bias onto neighboring lattice sites, provided the target site is empty. Even its most-studied onedimensional version, which describes driven single-file diffusion, exhibits rich phenomena, in particular, boundaryinduced phase transitions [3-7] and has a wide range of applications in different branches of physics. Experiments probing single-file diffusion have been performed with molecules in zeolites [8], colloidal particles in confined geometry [9], or optical lattices [10], and very recently with granular systems [11]. Driven single-file transport has been observed in biological systems, and the ASEP serves as a starting point for modeling the motion of ribosomes along the m-RNA during protein synthesis [12,13] and of molecular motors along microtubuli or actin filaments [14]. The ASEP is also at the heart of the cellular automaton approach to vehicular traffic on road networks $[15,16]$. In this setting, some predictions from the theory of boundary-induced phase transitions for the development of traffic jams have been verified empirically despite the complexity of real vehicular traffic [17]. Phenomena analogous to traffic jams have also been observed in the biological transport systems referred to above.

In the hydrodynamic approach to traffic flow [16] using partial differential equations, traffic jams correspond to shock solutions. A shock is a density discontinuity on the moving with some deterministic speed, determined by mass conservation. It is therefore no surprise that on the macroscopic Euler scale the time evolution of the particle density of the ASEP is described by the inviscid Burgers equation $[18,19]$, which develops shocks for generic initial data. With a view on applications of the ASEP to systems for which a hydrodynamic description is too coarse grained, it would thus be of interest to understand what fluctuating microscopic structure (on lattice scale) is underlying the phenomenon of shocks.

\footnotetext{
*Email address: f.tabatabaei@fz-juelich.de

†Email address: g.schuetz@fz-juelich.de
}

In fact, a great deal is known about shocks in the ASEP due to the exact solubility of the model. In the stationary regime, the shock structure has been studied as seen from a so-called second-class particle that serves as microscopic marker of the shock position. The particle density decays away from the shock exponentially (on lattice scale) to the respective constant bulk values $\rho_{1,2}$ of the two branches of the shock [20-22]. The shock position itself has been proved to perform Brownian motion on coarse grained diffusive scale [23]. For a particular strength of the driving field, the associated decay constant of the particle density vanishes, corresponding to a "minimal" intrinsic shock width. For this special value of the driving field, also the motion of the shock simplifies greatly. It performs a biased random walk on the microscopic lattice scale with explicitly known hopping rates $[24,25]$.

It is natural to ask whether this special feature of the ASEP survives in more complicated models of driven diffusive systems. In particular, one would like to investigate exclusion processes with nonconserved internal degrees of freedom, where particles may have different velocities, charges, masses, or other distinguishing properties that they can gain or lose (e.g., in a collision or chemical reaction). Here, we address this question in the simplest case of two possible internal states that each particle can possess. Such models have been investigated recently for various biological and vehicular transport phenomena [14,26,27]. Studying the microstructure of a shock illuminates the role of finite-size effects in first-order boundary-induced phase transitions that are associated with the motion of traffic jams [28-30] in finite systems.

The paper is organized as follows: In Sec. II, we determine the families of three-states models with pair interaction and a single conservation law. We also define shock measures for these systems. In Sec. III, we study exclusion processes with binary internal degree of freedom that allows for special traveling shock solutions on the finite lattice. In Sec. IV, we summarize our results and draw some conclusions.

\section{THREE-STATES PROCESSES WITH ONE CONSERVATION LAW}

\section{A. Stochastic dynamics}

On an abstract level, the exclusion process with a binary internal degree of freedom is a three-state process where the 
state of the system at any given time is described by a set of "occupation numbers" $\underline{n}=n_{1}, \ldots, n_{L}$ where $n_{k}=0,1,2$ is the local occupation number at site $k$ and $L$ is the number of sites. In Sec. III, we assign state 0 to an empty lattice site, state 1 to a particle of type $A$ and state 2 to a particle of type $B$. The labels $A$ and $B$ represent two possible internal states of a particle. However, in this section we first keep the treatment general and consider the occupation numbers as abstract objects, labeling one out of three possible states of a lattice site.

The bulk stochastic dynamics are defined by nearestneighbor transitions between the occupation variables, which occur independently and randomly in continuous time after an exponentially distributed waiting time. The mean $\tau\left(n_{k}^{\prime}, n_{k+1}^{\prime} ; n_{k}, n_{k+1}\right)$ of this waiting time depends on the transition $\left(n_{k}, n_{k+1}\right) \rightarrow\left(n_{k}^{\prime}, n_{k+1}^{\prime}\right)$, its inverse is the transition rate.

At the boundary sites $1, L$ we assume the system to be connected to some external reservoir with which the system can exchange particles. For injection and extraction of particles at the left boundary, we introduce the rates

$$
\begin{aligned}
& 0 \rightleftharpoons 1 \quad \alpha_{1}, \gamma_{1}, \\
& 0 \rightleftharpoons 2 \quad \alpha_{2}, \gamma_{2}, \\
& 1 \rightleftharpoons 2 \quad \alpha_{3}, \gamma_{3},
\end{aligned}
$$

and for the right boundary

$$
\begin{array}{ll}
0 \rightleftharpoons 1 & \delta_{1}, \beta_{1}, \\
0 \rightleftharpoons 2 & \delta_{2}, \beta_{2}, \\
1 \rightleftharpoons 2 & \delta_{3}, \beta_{3} .
\end{array}
$$

Here and below, the left rate refers to the process going from left to right, whereas the right rate is for the reversed process.

The time evolution defined above can be written in terms of a continuous-time master equation for the probability vector

$$
|P(t)\rangle=\sum_{\underline{n}} P\left(n_{1}, \ldots, n_{L} ; t\right)|\underline{n}\rangle,
$$

where $P\left(n_{1}, \ldots, n_{L} ; t\right)$ is the distribution for the probability of finding particles at sites 1 to $L$ and $|\underline{n}\rangle$ is the basis vector in the space of configurations in the naturally defined tensor basis [2]. The probability vector is normalized such that $\langle s \mid P\rangle=1$ with the summation vector $\langle s|=\Sigma_{\underline{n}}\langle\underline{n}|$ and scalar product $\left\langle\underline{n} \mid \underline{n}^{\prime}\right\rangle=\delta_{\underline{n}, \underline{n}^{\prime}}$. The time evolution is generated by the stochastic "quantum Hamiltonian" $H$ whose off diagonal matrix elements $H_{\underline{n}, \underline{n}}$ are the negative transition rates between configurations. As required by conservation of probability, the diagonal elements are the negative sum of transition rates in the respective column.

Therefore, the master equation is now described by the imaginary time Schrödinger equation

$$
\frac{d}{d t}|P(t)\rangle=-H|P(t)\rangle
$$

with the formal solution

$$
|P(t)\rangle=\mathrm{e}^{-H t}|P(0)\rangle .
$$

Because only nearest-neighbour interactions are included, the quantum Hamiltonian $H$ defined above has the structure

$$
H=b_{1}+\sum_{k=1}^{L-1} h_{k, k+1}+b_{L} \text {. }
$$

The local bulk transition matrix $h_{k, k+1}$ acts nontrivially only on sites $k$ and $k+1$. To define its matrix elements, we introduce an integer label

$$
i=3 n_{k}+n_{k+1}+1
$$

in the range $1 \leq i \leq 9$ for the occupation variables on two neighboring sites. The off-diagonal matrix elements $\left(h_{k, k+1}\right)^{(i j)}$ are then the transition rates $-w_{i j}$. Here, $i=3 n_{k}^{\prime}$ $+n_{k+1}^{\prime}+1$ labels the target configuration and $j$ is the respective label of the initial configuration $\left(n_{k}, n_{k+1}\right)$. The matrix elements of the boundary matrices $b_{1}$ and $b_{L}$ contain the boundary transition rates (1) and (2).

\section{B. Symmetries and conservation laws}

Within this setting, one could describe 72 different bulk transitions, corresponding to the 72 mathematically possible changes of configurations on a pair of sites. However, we shall reduce this large number by imposing various physically motivated constraints. First, we require a local conservation law. Generally, the physical interpretation of the conservation law depends on the physical interpretation of the occupation numbers $n_{k}$ and will become clear below. Mathematically, this means that in a periodic system some function $\Sigma_{k} C\left(n_{k}\right)$ of the local occupation numbers should remain invariant under the stochastic dynamics, i.e.,

$$
C\left(n_{k}^{\prime}\right)+C\left(n_{k+1}^{\prime}\right)=C\left(n_{k}\right)+C\left(n_{k+1}\right)
$$

for any local transition between configurations $i, j$. This constraint forces a large number of transition rates $w_{i j}$ to vanish. Physically, $C(n)$ is some observable property (such as mass or charge) of the state $n$.

The conservation condition (8) does not uniquely define the function $C(n)$. In order to analyze these constraints, we set $C(0)=0$ and $C(1)=1$. This involves no loss of generality since adding a constant to $C(n)$ or multiplying $C(n)$ by an arbitrary factor leaves (8) invariant. From inspection of (8) one can then see that there are three distinct families of solutions: (i) degenerate case, represented by $C(2)=C(1)=1$ (or equivalently $C(2)=C(0)=0$ ), (ii) linear nondegenerate case, represented by $C(2)=2$ (or equivalently $C(2)=-1$, $C(2)=1 / 2$ ), (iii) two independent conservation laws, represented by any other value of $C(2)$. The nondegenerate linear conservation law is treated elsewhere [33], the case of two conservation laws was studied in detail in $[31,32]$. Here, we investigate the degenerate conservation law. The degenerate function $C(n)$ has a natural interpretation as counting the number of particles at a given site irrespective of its internal state. This is the motivation behind the assignment of the state labels $A, B$ used below. 
The presence of a conservation law implies a lattice continuity equation

$$
\frac{d}{d t} C_{k}=j_{k-1}-j_{k}
$$

for the expectation $C_{k}=\left\langle C\left(n_{k}\right)\right\rangle$. This quantity plays the role of a local order parameter. The quantity $j_{k}$ is the current associated with the conservation law. It is given by the expectation of some combination of local occupation numbers, depending on the model under investigation; see below. Because we do not study here periodic systems, we do not require the boundary sites where the system is connected to the reservoir to respect the conservation law. The quantities $j_{0}, j_{L}$ entering the continuity equation for $k=1$ and $k=L$, respectively, are source terms resulting from the reservoirs. They are functions of the reservoir densities.

Second, in addition to the conservation law, we require $P T$ invariance (i.e., the bulk dynamics should be symmetric under combined time reversal and space reflection). This physical input generalizes the equilibrium condition of detailed balance to allow for external driving forces, which lead to a bias in the hopping rates. In such a case, the system is forced into a nonequilibrium steady state with a stationary current flowing in the system. Well-known examples for models of this kind are exclusion processes satisfying pairwise balance [34]. $P T$ invariance is implemented by demanding detailed balance with respect to the space-reflected target state of a local transition. As a result, there are pairwise relations between some of the 72 transition rates, see below.

\section{Product measures}

Even though the number of independent model parameters is greatly reduced by particle conservation and $P T$ symmetry, the form of the stationary distribution is not determined by these constraints. In order to be able to carry out explicit computations, we restrict ourselves to systems such that the stationary distribution of the stochastic dynamics factorizes, i.e., one has a product measure. In the quantum Hamiltonian formalism introduced above, a product measure is given by a tensor product

$$
\left.\left.\left.|P\rangle=\mid P_{1}\right) \otimes \mid P_{2}\right) \otimes \cdots \otimes \mid P_{L}\right) .
$$

Here, the three-component single-site probability vectors $\left.\mid P_{k}\right)$ has as components the probabilities $P\left(n_{k}\right)$ of finding state $n$ at site $k$. In the stationary distribution, these probabilities are position independent, $\left.\left(P_{k}\right) \equiv \mid P\right)$, and the stationary probability vector thus has the homogeneous product form

$$
\left.\left|P^{*}\right\rangle=\mid P\right)^{\otimes L} .
$$

We represent the single-site basis vectors for this model as

$$
\left.\left.\mid 0)=\left(\begin{array}{l}
1 \\
0 \\
0
\end{array}\right), \quad \mid A\right)=\left(\begin{array}{l}
0 \\
1 \\
0
\end{array}\right), \quad \mid B\right)=\left(\begin{array}{l}
0 \\
0 \\
1
\end{array}\right)
$$

and parametrize the stationary one-site marginal

$$
P)=\frac{1}{\nu}\left(\begin{array}{c}
1 \\
z \\
c z
\end{array}\right)
$$

by a fugacity $z$ and the ratio $c$ of $A$ and $B$ concentrations. The normalization factor

$$
\nu=1+z+c z
$$

is the local partition function. Thus, one has for this grand canonical ensemble

$$
\rho^{A}=\frac{z}{\nu}, \quad \rho^{B}=c \frac{z}{\nu}
$$

and for the total conserved particle density

$$
\rho:=\rho^{A}+\rho^{B}=z \frac{d}{d z} \ln \nu=(1+c) \frac{z}{\nu} .
$$

In formal analogy to systems in thermal equilibrium, we shall refer to the logarithm of the fugacity as chemical potential.

By definition of stationarity the stationary probability vector satisfies the eigenvalue equation

$$
H\left|P^{*}\right\rangle=0 .
$$

Requiring the existence of a stationary product measure imposes constraints both on the bulk rates and on the boundary rates, which fix the bulk fugacity $z$. Once these conditions are determined the model is fully defined and its stationary distribution is given. Note that, by definition, a stationary product measure has no correlations between the occupation numbers at different sites.

After defining the model in this way, we shall relax some of the constraints on the boundary conditions and study the time evolution of (nonstationary) shock measures of the form

$$
\left.\left.|k\rangle=\mid P_{1}\right)^{\otimes k} \otimes \mid P_{2}\right)^{\otimes L-k} .
$$

These shock measures have single-site probabilities given by a fugacity $z_{1}$ in the left chain segment up to site $k$ and fugacity $z_{2}$ in the remaining chain segment from site $L-k$ up to site $L$. Such a shock measure fully defines the internal structure of the shock. Because there are no correlations in a shock measure, one may regard the lattice unit as the intrinsic shock width. A typical configuration has a sharp decrease of the mean interparticle distance across the lattice point $k$. The boundary fugacities of the system are chosen such that each chain segment is stationary at its boundary. The measure itself, however, is not stationary for $z_{1} \neq z_{2}$. The associated gradient of the chemical potential together with external driving forces entering the bulk hopping rates drive the system into an nonequilibrium steady state, to be determined below as the final stage of the time evolution of the shock measure.

\section{EXCLUSION PROCESS WITH BINARY INTERNAL DEGREE OF FREEDOM}

We now implement the constraints discussed above. The degenerate conservation law (8) forces 48 transition rates to vanish. The following 24 transitions remain: 


$$
\begin{aligned}
& 0 A \rightarrow A 0 w_{42}, \quad A 0 \rightarrow 0 A w_{24}, \\
& 0 B \rightarrow B 0 w_{73}, \quad B 0 \rightarrow 0 B w_{37}, \\
& A B \rightarrow B A w_{86}, \quad B A \rightarrow A B w_{68}, \\
& B 0 \rightarrow A 0 w_{47}, \quad 0 A \rightarrow 0 B w_{32}, \\
& 0 B \rightarrow A 0 w_{43}, \quad 0 A \rightarrow B 0 w_{72}, \\
& A 0 \rightarrow B 0 w_{74}, \quad 0 B \rightarrow 0 A w_{23}, \\
& B 0 \rightarrow 0 A w_{27}, \quad A 0 \rightarrow 0 B w_{34}, \\
& B A \rightarrow A A w_{58}, \quad A A \rightarrow A B w_{65}, \\
& A B \rightarrow A A w_{56}, \quad A A \rightarrow B A w_{85}, \\
& B B \rightarrow A A w_{59}, \quad A A \rightarrow B B w_{95}, \\
& B B \rightarrow B A w_{89}, \quad A B \rightarrow B B w_{96}, \\
& B B \rightarrow A B w_{69}, \quad B A \rightarrow B B w_{98} .
\end{aligned}
$$

Parity-time invariance leads to pairwise relations between some of these rates. Time reversal symmetry means to have detailed balance $p^{*}(n) w\left(n \rightarrow n^{\prime}\right)=p^{*}\left(\underline{n}^{\prime}\right) w\left(\underline{n}^{\prime} \rightarrow n\right)$. In order to combine this relation with the parity (space reflection) operation, we change the position of neighboring sites with each other in the initial configuration and final configuration on the left-hand side of the detailed-balance relation. Using (15), this yields the following symbolic relations for the rates:

$$
w(A \rightarrow B)=c w(B \rightarrow A)
$$

for each particle on a pair of neighboring sites. In order to illustrate how this equation leads to some relations between rates, we calculate one of them explicitly. For example, the reaction process $A B \rightarrow A A$ with the rate $w_{56}$ changes to $A A$ $\rightarrow A B$ after applying time reversal symmetry and by operating space reflection one obtains $A A \rightarrow B A$ with the rate $w_{86}$. By using (20) for every single site, we obtain $w_{85}=c w_{56}$.

With the relation (20), we can reduce the number of independent rates in the process (19) to only 15 nonstationary rates (viz. six hopping rates and nine "reaction rates" for changes of the internal states of the particles). For clarity, we represent all of the hopping rates by $h$ 's and reaction process by $r$ 's and write the rates as

$$
\begin{array}{ll}
w_{47}=r_{1}, & w_{32}=c r_{1}, \\
w_{43}=r_{2}, & w_{72}=c r_{2}, \\
w_{23}=r_{3}, & w_{74}=c r_{3}, \\
w_{27}=r_{4}, & w_{34}=c r_{4}, \\
w_{58}=r_{5}, & w_{65}=c r_{5},
\end{array}
$$

$$
\begin{aligned}
& w_{56}=r_{6}, \quad w_{85}=c r_{6}, \\
& w_{59}=r_{7}, \quad w_{95}=c^{2} r_{7}, \\
& w_{89}=r_{8}, \quad w_{96}=c r_{8}, \\
& w_{69}=r_{9}, \quad w_{98}=c r_{9}, \\
& w_{42}=h_{1}, \quad w_{73}=h_{2}, \\
& w_{24}=h_{3}, \quad w_{86}=h_{4}, \\
& w_{68}=h_{5}, \quad w_{37}=h_{6} .
\end{aligned}
$$

Note that for the case $w_{95}$, because in $A A \rightarrow B B$ both of the particles at two neighboring sites are changing, the factor $c$ appears twice.

In the quantum Hamiltonian formalism, the bulk transition matrix is then given by

$$
h_{k, k+1}=-\left(\begin{array}{ccccccccc}
. & 0 & 0 & 0 & 0 & 0 & 0 & 0 & 0 \\
0 & . & r_{3} & h_{3} & 0 & 0 & r_{4} & 0 & 0 \\
0 & c r_{1} & . & c r_{4} & 0 & 0 & h_{6} & 0 & 0 \\
0 & h_{1} & r_{2} & . & 0 & 0 & r_{1} & 0 & 0 \\
0 & 0 & 0 & 0 & . & r_{6} & 0 & r_{5} & r_{7} \\
0 & 0 & 0 & 0 & c r_{5} & . & 0 & h_{5} & r_{9} \\
0 & c r_{2} & h_{2} & c r_{3} & 0 & 0 & . & 0 & 0 \\
0 & 0 & 0 & 0 & c r_{6} & h_{4} & 0 & . & r_{8} \\
0 & 0 & 0 & 0 & c^{2} r_{7} & c r_{8} & 0 & c r_{9} & .
\end{array}\right)_{k, k+1}
$$

and the boundary transition matrices have the forms

$$
\begin{gathered}
b_{1}=-\left(\begin{array}{ccc}
-\left(\alpha_{1}+\alpha_{2}\right) & \gamma_{1} & \gamma_{2} \\
\alpha_{1} & -\left(\gamma_{1}+\alpha_{3}\right) & \gamma_{3} \\
\alpha_{2} & \alpha_{3} & -\left(\gamma_{2}+\gamma_{3}\right)
\end{array}\right)_{1}, \\
b_{L}=-\left(\begin{array}{ccc}
-\left(\delta_{1}+\delta_{2}\right) & \beta_{1} & \beta_{2} \\
\delta_{1} & -\left(\beta_{1}+\delta_{3}\right) & \beta_{3} \\
\delta_{2} & \beta_{3} & -\left(\beta_{2}+\beta_{3}\right)
\end{array}\right)_{L} .
\end{gathered}
$$

\section{A. Product measure}

With (13), the homogeneous product measure has the form

$$
\left|P^{*}\right\rangle=\frac{1}{\nu^{L}}\left(\begin{array}{c}
1 \\
z \\
c z
\end{array}\right)^{\otimes L} .
$$

It is convenient to define

$$
\hat{h}_{i, i+1}=h_{i, i+1}-\left[E\left(\hat{n}_{i}^{A}-\hat{n}_{i+1}^{A}\right)+E^{\prime}\left(\hat{n}_{i}^{B}-\hat{n}_{i+1}^{B}\right)\right],
$$

where $E, E^{\prime}$ are arbitrary constants and $\hat{n}_{A}$ and $\hat{n}_{B}$ are number operators with eigenvalue 1 if a particle of the respective 
species is present and 0 otherwise. Furthermore, we define modified boundary matrices

$$
\hat{b}_{1}=b_{1}+E \hat{n}_{1}^{A}+E^{\prime} \hat{n}_{1}^{B}, \quad \hat{b}_{L}=b_{L}-E \hat{n}_{L}^{A}-E^{\prime} \hat{n}_{L}^{B} .
$$

This allows us to rewrite the quantum Hamiltonian as

$$
H=\hat{b}_{1}+\sum_{i=1}^{L-1} \hat{h}_{i, i+1}+\hat{b}_{L}
$$

The eigenvalue equation (17) may be rewritten

$$
0=\hat{h}_{i, i+1}\left|P^{*}\right\rangle=\left(\hat{b}_{1}+g\right)\left|P^{*}\right\rangle=\left(\hat{b}_{L}-g\right)\left|P^{*}\right\rangle,
$$

with a further arbitrary constant $g$.

This trick allows us to determined the conditions on the rates that ensure that (25) is actually stationary. For the bulk rates, (29) yields

$$
\begin{gathered}
E=h_{3}-h_{1}+c\left(r_{3}+r_{4}-r_{1}-r_{2}\right), \\
E^{\prime}=h_{6}-h_{2}+r_{1}+r_{4}-r_{2}-r_{3} .
\end{gathered}
$$

Furthermore, some algebra shows that the bulk rates must satisfy the following condition for stationarity:

$$
\begin{aligned}
& h_{6}-h_{2}+h_{1}-h_{3}+h_{4}-h_{5}+(1+c)\left(r_{1}-r_{3}\right)+(1-c)\left(r_{4}-r_{2}\right) \\
& \quad+c\left(r_{8}-r_{9}\right)+r_{6}-r_{5}=0 .
\end{aligned}
$$

In order to satisfy the eigenvalue equation (29) at the boundaries the terms involving $E, E^{\prime}$ must cancel. For the left boundary, this yields the two relations

$$
\begin{aligned}
& {\left[h_{1}-h_{3}+c\left(2\left(r_{2}-r_{4}\right)+h_{2}-h_{6}\right)\right] z} \\
& \quad=\gamma_{1} z \nu+\gamma_{2} c z \nu-\left(\alpha_{1}+\alpha_{2}\right) \nu \\
& \quad=-\beta_{1} z \nu-\beta_{2} c z \nu+\left(\delta_{1}+\delta_{2}\right) \nu,
\end{aligned}
$$

and similarly at the right boundary

$$
\begin{aligned}
& {\left[\left(-r_{5}+r_{6}+c\left(r_{8}-r_{9}\right)+h_{4}-h_{5}\right) z-r_{1}+r_{2}+r_{3}-r_{4}+h_{2}-h_{6}\right] c z} \\
& \quad=\left(\gamma_{2}+\gamma_{3}\right) c z \nu-\alpha_{2} \nu-\alpha_{3} z(1+z) \nu \\
& \quad=-\left(\beta_{2}+\beta_{3}\right) c z \nu+\delta_{2} \nu+\delta_{3} z(1+z) \nu .
\end{aligned}
$$

These relations define a model for which the product measure with constant fugacity $z$ is stationary. The fugacity is determined by its boundary value encoded in the boundary rates.

\section{B. Fugacity gradient}

Now we generalize the model to allow for different fugacities $z_{1}, z_{2}$ at the two boundaries. The product measure is then no longer stationary, and there is no general principle that would constrain the form of the stationary distribution. However, in principle, its properties can be calculated from the studying the time evolution of the system starting from some initial distribution.

In general, solving for the dynamics of a many-particle system is a much harder task than determining its stationary distribution. However, guided by previous experience [25], we make as ansatz an initial distribution, which is a shock

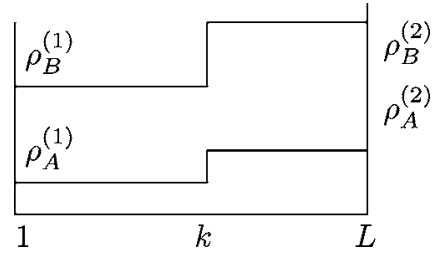

FIG. 1. Coarse-grained density profiles of a shock measure with shock between sites $k, k+1$.

measure connecting the two boundary fugacities. The representation of the shock measure here is

$$
|k\rangle=\frac{1}{\nu_{1}^{k} \nu_{2}^{(L-k)}}\left(\begin{array}{c}
1 \\
z_{1} \\
c z_{1}
\end{array}\right)^{\otimes k} \otimes\left(\begin{array}{c}
1 \\
z_{2} \\
c z_{2}
\end{array}\right)^{\otimes L-k} .
$$

On a coarse-grained scale, the density profile corresponding to this measure has a jump discontinuity; see Fig. 1. We search for conditions on the rates such that

$$
\frac{d}{d t}|k\rangle=d_{1}|k-1\rangle+d_{2}|k+1\rangle-\left(d_{1}+d_{2}\right)|k\rangle .
$$

This implies that the family of shock measures labelled by the shock position $k$ is closed under the time evolution of the many-particle system. Physically, this behavior corresponds to a random walk of the shock with hopping rates $d_{1},\left(d_{2}\right)$ to the left (right).

In order to have the random walk equation (35) for the shock, one replaces the left-hand side by the (negative) quantum Hamiltonian in the form (28). Then in each branch of the shock, one has $\hat{h}_{i, i+1}|k\rangle=0$, except for $i=k$. Stationarity at the boundaries implies

$$
\begin{gathered}
b_{1}\left|P^{*}\right\rangle=\left(-E \hat{n}_{1}^{A}-E^{\prime} \hat{n}_{1}^{B}+g_{1}\right)\left|P^{*}\right\rangle, \\
b_{L}\left|P^{*}\right\rangle=\left(E \hat{n}_{L}^{A}+E^{\prime} \hat{n}_{L}^{B}-g_{2}\right)\left|P^{*}\right\rangle,
\end{gathered}
$$

which leads to

$$
\begin{gathered}
g_{1}=E \frac{z_{1}}{\nu_{1}}+E^{\prime} \frac{c z_{1}}{\nu_{1}}=\alpha_{1}+\alpha_{2}-\left(\gamma_{1}+c \gamma_{2}\right) z_{1}, \\
g_{2}=E \frac{z_{2}}{\nu_{2}}+E^{\prime} \frac{c z_{2}}{\nu_{2}}=-\left(\delta_{1}+\delta_{2}\right)+\left(\beta_{1}+c \beta_{2}\right) z_{2} .
\end{gathered}
$$

The random walk condition for the shock thus leads to nine equations

$$
\left(-\hat{h}_{k, k+1}+d_{1}+d_{2}-g_{1}+g_{2}\right)|k\rangle-d_{1}|k-1\rangle-d_{2}|k+1\rangle=0
$$

for the bulk rates. Three of these conditions are fulfilled without any constraint on the rates, leaving the following six equations:

$$
S-d_{1} \frac{\nu_{1}}{\nu_{2}}-d_{2} \frac{\nu_{2}}{\nu_{1}}=0
$$




$$
\begin{gathered}
\left(c r_{4}+h_{3}\right)\left(z_{1}-z_{2}\right)+S z_{2}-d_{1} z_{2} \frac{\nu_{1}}{\nu_{2}}-d_{2} z_{1} \frac{\nu_{2}}{\nu_{1}}=0, \\
\left(r_{4}+h_{6}\right)\left(z_{1}-z_{2}\right)+S z_{2}-d_{1} z_{2} \frac{\nu_{1}}{\nu_{2}}-d_{2} z_{1} \frac{\nu_{2}}{\nu_{1}}=0, \\
\left(c r_{2}+h_{1}\right)\left(z_{2}-z_{1}\right)+S z_{1}-d_{1} z_{2} \frac{\nu_{1}}{\nu_{2}}-d_{2} z_{1} \frac{\nu_{2}}{\nu_{1}}=0, \\
S-d_{1} \frac{z_{2} \nu_{1}}{z_{1} \nu_{2}}-d_{2} \frac{z_{1} \nu_{2}}{z_{2} \nu_{1}}=0, \\
\left(r_{2}+h_{2}\right)\left(z_{2}-z_{1}\right)+S z_{1}-d_{1} z_{2} \frac{\nu_{1}}{\nu_{2}}-d_{2} z_{1} \frac{\nu_{2}}{\nu_{1}}=0,
\end{gathered}
$$

where

$$
S=d_{1}+d_{2}+g_{2}-g_{1} .
$$

Solving Eqs. (41)-(46) leads to three independent relations between bulk rates and densities

$$
\begin{gathered}
h_{3}+c r_{4}=h_{6}+r_{4} \equiv p, \\
h_{1}+c r_{2}=h_{2}+r_{2} \equiv q, \\
\frac{p}{q}=\frac{z_{2}}{z_{1}},
\end{gathered}
$$

and two relations

$$
\begin{aligned}
& d_{1}=q \frac{\nu_{2}}{\nu_{1}}, \\
& d_{2}=p \frac{\nu_{1}}{\nu_{2}},
\end{aligned}
$$

which express the shock hopping rates in terms of the hopping rates of the model and the fugacities of the shock. On this parameter manifold, the stationarity condition (31) reduces to

$$
h_{4}-h_{5}+(1+c)\left(r_{1}-r_{3}\right)+c\left(r_{8}-r_{9}\right)+r_{6}-r_{5}=0 \text {. }
$$

The shock performs a random walk for a specific ratio of the boundary fugacities or, equivalently, at some specific strength of the driving force encoded in the particle hopping rates. Thus, shock mean velocity $v_{s}$ in terms of vacancy density and hopping rates is

$$
v_{s}=\frac{q \nu_{2}^{2}-p \nu_{1}^{2}}{\nu_{1} \nu_{2}},
$$

and its diffusion coefficient as long as the shock is far from the boundaries is

$$
D_{s}=\frac{p \nu_{1}^{2}+q \nu_{2}^{2}}{2 \nu_{1} \nu_{2}} \text {. }
$$

Equation (35) is valid for $k \neq 0, L+1$. For $k=0$ and $k=L$ +1 the boundary relations (32) and (33) give rise to reflective boundary for the shock motion. Therefore, from the shock hopping rates and its biased random walk dynamics we can read off the stationary distribution of the system for different boundary densities. This is a linear combination of shock measures

$$
\left|P^{*}\right\rangle \propto \sum_{k}\left(\frac{d_{1}}{d_{2}}\right)^{k}|k\rangle .
$$

For $d_{1}>d_{2}$ (bias to the right), the stationary shock position is in the vicinity of the right boundary, leaving the system in a phase of low density. Conversely, for $d_{1}<d_{2}$, the system is in a high-density phase. At $d_{1}=d_{2}$ the system undergoes a firstorder nonequilibrium transition [6]. Here, the shock has no bias and can be found with equal probability anywhere on the lattice. The stationary density profile is linear, but a typical particle configuration has two different regions of constant (but fluctuating) density. The density jumps quickly from one density to another in some small region of the lattice.

\section{Steady-state current}

In order to make contact with the ASEP, we calculate the stationary current for this model. In order to identify the current, we first calculate the equation of motion for the expected local particle densities,

$$
\begin{aligned}
\frac{d}{d t}\left\langle n_{k}^{A}\right\rangle= & -\left(h_{1}+c r_{1}+c r_{2}\right)\left\langle n_{k-1}^{0} n_{k}^{A}\right\rangle+h_{3}\left\langle n_{k-1}^{A} n_{k}^{0}\right\rangle+\left(h_{4}-r_{6}\right)\left\langle n_{k-1}^{A} n_{k}^{B}\right\rangle-\left(h_{5}+c r_{9}\right)\left\langle n_{k-1}^{B} n_{k}^{A}\right\rangle+r_{3}\left\langle n_{k-1}^{0} n_{k}^{B}\right\rangle+r_{4}\left\langle n_{k-1}^{B} n_{k}^{0}\right\rangle-c\left(r_{5}+c r_{7}\right) \\
& \times\left\langle n_{k-1}^{A} n_{k}^{A}\right\rangle+\left(r_{7}+r_{8}\right)\left\langle n_{k-1}^{B} n_{k}^{B}\right\rangle+h_{1}\left\langle n_{k}^{0} n_{k+1}^{A}\right\rangle-\left(h_{3}+c r_{3}+c r_{4}\right)\left\langle n_{k}^{A} n_{k+1}^{0}\right\rangle-\left(h_{4}+c r_{8}\right)\left\langle n_{k}^{A} n_{k+1}^{B}\right\rangle+\left(h_{5}+r_{5}\right)\left\langle n_{k}^{B} n_{k+1}^{A}\right\rangle \\
& +r_{1}\left\langle n_{k}^{B} n_{k+1}^{0}\right\rangle-r_{2}\left\langle n_{k}^{0} n_{k+1}^{B}\right\rangle-c\left(r_{6}+c r_{7}\right)\left\langle n_{k}^{A} n_{k+1}^{A}\right\rangle+\left(r_{7}+r_{9}\right)\left\langle n_{k}^{B} n_{k+1}^{B}\right\rangle, \\
\frac{d}{d t}\left\langle n_{k}^{B}\right\rangle= & -\left(h_{2}+r_{2}+r_{3}\right)\left\langle n_{k-1}^{0} n_{k}^{B}\right\rangle+h_{6}\left\langle n_{k-1}^{B} n_{k}^{0}\right\rangle-\left(h_{4}+r_{6}\right)\left\langle n_{k-1}^{A} n_{k}^{B}\right\rangle+\left(h_{5}+c r_{9}\right)\left\langle n_{k-1}^{B} n_{k}^{A}\right\rangle+c r_{1}\left\langle n_{k-1}^{0} n_{k}^{A}\right\rangle+c r_{4}\left\langle n_{k-1}^{A} n_{k}^{0}\right\rangle+c\left(r_{5}+c r_{7}\right) \\
& \times\left\langle n_{k-1}^{A} n_{k}^{A}\right\rangle-\left(r_{7}+r_{8}\right)\left\langle n_{k-1}^{B} n_{k}^{B}\right\rangle+h_{2}\left\langle n_{k}^{0} n_{k+1}^{B}\right\rangle-\left(h_{6}+r_{1}+r_{4}\right)\left\langle n_{k}^{B} n_{k+1}^{0}\right\rangle+\left(h_{4}+c r_{8}\right)\left\langle n_{k}^{A} n_{k+1}^{B}\right\rangle-\left(h_{5}+r_{5}\right)\left\langle n_{k}^{B} n_{k+1}^{A}\right\rangle \\
& +c r_{2}\left\langle n_{k}^{0} n_{k+1}^{A}\right\rangle+c r_{3}\left\langle n_{k}^{A} n_{k+1}^{0}\right\rangle+c\left(r_{6}+c r_{7}\right)\left\langle n_{k}^{A} n_{k+1}^{A}\right\rangle-\left(r_{7}+r_{9}\right)\left\langle n_{k}^{B} n_{k+1}^{B}\right\rangle .
\end{aligned}
$$

This can be written in terms of $A$ and $B$ particle current 


$$
\begin{aligned}
& \frac{d}{d t}\left\langle n_{k}^{A}\right\rangle=j_{k-1}^{A}-j_{k}^{A}+S_{k}, \\
& \frac{d}{d t}\left\langle n_{k}^{B}\right\rangle=j_{k-1}^{B}-j_{k}^{B}-S_{k},
\end{aligned}
$$

where the source term

$$
\begin{aligned}
S_{k}= & \left(c r_{1}-\frac{c r_{2}}{2}\right)\left\langle n_{k-1}^{0} n_{k}^{A}\right\rangle+\left(\frac{r_{2}}{2}+r_{3}\right)\left\langle n_{k-1}^{0} n_{k}^{B}\right\rangle+\frac{r_{4}}{2}\left\langle n_{k-1}^{B} n_{k}^{0}\right\rangle-\frac{c r_{4}}{2}\left\langle n_{k-1}^{A} n_{k}^{0}\right\rangle-\left(c r_{5}+c^{2} r_{7}\right)\left\langle n_{k-1}^{A} n_{k}^{A}\right\rangle+r_{6}\left\langle n_{k-1}^{A} n_{k}^{B}\right\rangle \\
& +\left(r_{7}+r_{8}\right)\left\langle n_{k-1}^{B} n_{k}^{B}\right\rangle-c r_{9}\left\langle n_{k-1}^{B} n_{k}^{A}\right\rangle+\left(r_{1}+\frac{r_{4}}{2}\right)\left\langle n_{k}^{B} n_{k+1}^{0}\right\rangle+\frac{r_{2}}{2}\left\langle n_{k}^{0} n_{k+1}^{B}\right\rangle-\frac{c r_{2}}{2}\left\langle n_{k}^{0} n_{k+1}^{A}\right\rangle-\left(c r_{3}+\frac{c r_{4}}{2}\right)\left\langle n_{k}^{A} n_{k+1}^{0}\right\rangle \\
& +r_{5}\left\langle n_{k}^{B} n_{k+1}^{A}\right\rangle-\left(c r_{6}+c^{2} r_{7}\right)\left\langle n_{k}^{A} n_{k+1}^{A}\right\rangle+\left(r_{7}+r_{9}\right)\left\langle n_{k}^{B} n_{k+1}^{B}\right\rangle-c r_{8}\left\langle n_{k}^{A} n_{k+1}^{B}\right\rangle
\end{aligned}
$$

expresses the fact that the individual particle densities are not conserved. The particle currents are given by the expectations

$$
\begin{aligned}
& j_{k}^{A}=-\left(h_{1}+\frac{c r_{2}}{2}\right)\left\langle n_{k}^{0} n_{k+1}^{A}\right\rangle+\left(h_{3}+\frac{c r_{4}}{2}\right)\left\langle n_{k}^{A} n_{k+1}^{0}\right\rangle+h_{4}\left\langle n_{k}^{A} n_{k+1}^{B}\right\rangle-h_{5}\left\langle n_{k}^{B} n_{k+1}^{A}\right\rangle-\frac{r_{2}}{2}\left\langle n_{k}^{0} n_{k+1}^{B}\right\rangle+\frac{r_{4}}{2}\left\langle n_{k}^{B} n_{k+1}^{0}\right\rangle, \\
& j_{k}^{B}=-\left(h_{2}+\frac{r_{2}}{2}\right)\left\langle n_{k}^{0} n_{k+1}^{B}\right\rangle+\left(h_{6}+\frac{r_{4}}{2}\right)\left\langle n_{k}^{B} n_{k+1}^{0}\right\rangle-h_{4}\left\langle n_{k}^{A} n_{k+1}^{B}\right\rangle+h_{5}\left\langle n_{k}^{B} n_{k+1}^{A}\right\rangle-\frac{c r_{2}}{2}\left\langle n_{k}^{0} n_{k+1}^{A}\right\rangle+\frac{c r_{4}}{2}\left\langle n_{k}^{A} n_{k+1}^{0}\right\rangle .
\end{aligned}
$$

By adding the two individual currents, we find the total particle current to be given by

$$
j_{k}=j_{k}^{A}+j_{k}^{B}=-h_{1}\left\langle n_{k}^{0} n_{k+1}^{A}\right\rangle+h_{3}\left\langle n_{k}^{A} n_{k+1}^{0}\right\rangle-h_{2}\left\langle n_{k}^{0} n_{k+1}^{B}\right\rangle+h_{6}\left\langle n_{k}^{B} n_{k+1}^{0}\right\rangle-r_{2}\left\langle n_{k}^{0} n_{k+1}^{B}\right\rangle-c r_{2}\left\langle n_{k}^{0} n_{k+1}^{A}\right\rangle+c r_{4}\left\langle n_{k}^{A} n_{k+1}^{0}\right\rangle+r_{4}\left\langle n_{k}^{B} n_{k+1}^{0}\right\rangle .
$$

In the steady state, we obtain

$$
j^{*}=\frac{h_{3}-h_{1}+c\left(h_{6}-h_{2}\right)+2 c\left(r_{4}-r_{2}\right)}{1+c} \rho(1-\rho),
$$

where $\rho$ is the average density (16). This can be written in terms of $E$ and $E^{\prime}$

$$
j^{*}=\frac{E+c E^{\prime}}{1+c} \rho(1-\rho) .
$$

This is the well-known parabolic current-density relation of the ASEP [1,2] where the density-independent prefactor plays the role of the hopping bias. In fact, on the special manifold which gives rise to the random walk of the shock we find, using (48) and (49), the simpler expression

$$
j^{*}=(p-q) \rho(1-\rho) .
$$

\section{CONCLUSIONS}

We have found that three-state lattice gases with a single local conservation law can be classified into two families, one where the conserved quantity is a linear function of the occupation variable and another where the function is degenerate, i.e., takes the same value for two different states. Nonlinear nondegenerate functions lead to two independently conserved quantities.
The degenerate linear conservation describes a class of asymmetric exclusion processes with a binary internal degree of freedom. We have identified constraints on the transition rates such that the stationary distribution is a product measure, parametrized by the nonequilibrium analog of the fugacity. For open systems with different boundary fugacities, we have found a complete list of models where the shock performs a biased random walk on the lattice. For these systems, we have detailed knowledge about the microscopic structure of the shock. As in other models studied previously (see [33], and references therein), these shocks are intrinsically maximally sharp and behave like collective single-particle excitations already on the lattice scale-not only after coarse-graining where all the microscopic features of the shock are lost. Apparently, this enormous reduction in the number of dynamical degrees of freedom in a subspace of the stochastic dynamics appears more frequently than previously suggested [35].

An immediate consequence of the random walk dynamics of the shock is the existence of a first-order boundaryinduced phase transition, which occurs if the boundary fugacities reverse the mean shock velocity. Away from this special manifold, our result for the sharpness of the shock suggest that finite systems with lattice size of the order 10 can be well described by the domain wall theory for firstorder boundary-induced phase transitions [6,7], with limitations analogous to those obtained from the exact results of Ref. [30]. 
It is intriguing that the maximal sharpness appears at some specific value of the driving force or, equivalently, ratio of boundary fugacities. It would be interesting to investigate whether such a field-induced sharpening of the interface is a special property of lattice models or can appear also in continuum systems, such as the recently studied mass transfer models $[36,37]$. It is also an open problem whether there can be an analogous reduction of the shock dynamics to a random walk problem in exclusion processes where the stationary distribution does not factorize [38,39].

\section{ACKNOWLEDGMENT}

F.T. would like to thank R.J. Harris for useful discussions.
[1] T. M. Liggett, Stochastic Interacting Systems: Voter, Contact and Exclusion Processes, (Springer, New York, 1999).

[2] G. M. Schütz, in: Phase Transitions and Critical Phenomena, Vol. 19, edited by C. Domb and J. Lebowitz (Academic, London, 2001).

[3] J. Krug, Phys. Rev. Lett. 67, 1882 (1991).

[4] G. Schütz and E. Domany, J. Stat. Phys. 72, 277 (1993).

[5] B. Derrida, M. R. Evans, V. Hakim, and V. Pasquier, J. Phys. A 26, 1493 (1993).

[6] A. B. Kolomeisky, G. M. Schütz, E. B. Kolomeisky, and J. P. Straley, J. Phys. A 31, 6911 (1998).

[7] V. Popkov and G. M. Schütz, Europhys. Lett. 48, 257 (1999).

[8] V. Kukla, J. Kornatowski, D. Demuth, I. Girnus, H. Pfeifer, L. V. C. Rees, S. Schunk, K. Unger, and J. Kärger, Science 272 , 702 (1996).

[9] Q-H. Wei, C. Bechinger, and P. Leiderer, Science 287, 625 (2000).

[10] C. Lutz, M. Kollmann, P. Leiderer, and C. Bechinger, J. Phys.: Condens. Matter 16, S4075 (2004).

[11] G. Coupier, M. S. Jean, and C. Guthmann, cond-mat/0603050.

[12] J. T. MacDonald, J. H. Gibbs, and A. C. Pipkin, Biopolymers 6, 1 (1968).

[13] G. M. Schütz, Int. J. Mod. Phys. B 11, 197 (1997).

[14] K. Nishinari, Y. Okada, A. Schadschneider, and D. Chowdhury, Phys. Rev. Lett. 95, 118101 (2005).

[15] D. Chowdhury, L. Santen, and A. Schadschneider, Phys. Rep. 329, 199 (2000).

[16] D. Helbing, Rev. Mod. Phys. 73, 1067 (2001).

[17] V. Popkov, L. Santen, A. Schadschneider, and G. M. Schütz, J. Phys. A 34, L45 (2001).

[18] J. M. Burgers, The Non Linear Diffusion Equation (Reidel, Boston, 1974).

[19] C. Kipnis and C. Landim, Scaling Limits of Interacting Particle Systems (Springer, New York, 1999).
[20] P. A. Ferrari, C. Kipnis, and E. Saada, Ann. Probab. 19, 226 (1991).

[21] B. Derrida, J. L. Lebowitz, and E. R. Speer, J. Stat. Phys. 89, 135 (1997).

[22] B. Derrida, S. Goldstein, J. L. Lebowitz, and E. R. Speer, J. Stat. Phys. 93, 547 (1998).

[23] P. A. Ferrari and L. R. G. Fontes, Probab. Theory Relat. Fields 99, 305 (1994).

[24] V. Belitsky and G. M. Schütz, Electron. J. Probab. 7, 11 (2002).

[25] K. Krebs, F. H. Jafarpour, and G. M. Schütz, New J. Phys. 5, 145 (2003).

[26] O. J. O’Loan, M. R. Evans, and M. E. Cates, Europhys. Lett. 42, 137 (1998); Phys. Rev. E 58, 1404 (1998).

[27] K. Nishinari, D. Chowdhury, and A. Schadschneider, Phys. Rev. E 67, 036120 (2003).

[28] M. Dudzinski and G. M. Schütz, J. Phys. A 33, 8351 (2000).

[29] Z. Nagy, C. Appert, and L. Santen, J. Stat. Phys. 109, 623 (2002).

[30] J. de Gier and F. H. L. Essler, Phys. Rev. Lett. 95, 240601 (2005).

[31] A. Rákos and G. M. Schütz, J. Stat. Phys. 117, 55 (2004).

[32] F. H. Jafarpour, Physica A 358, 413 (2005).

[33] F. Tabatabaei and G. M. Schütz, Diff. Fund. 4, 5.1 (2006).

[34] G. M. Schütz, R. Ramaswamy, and M. Barma, J. Phys. A 29, 837 (1996).

[35] M. Balazs, J. Stat. Phys. 105, 511 (2001).

[36] M. R. Evans, S. N. Majumdar, and R. K. P. Zia, J. Phys. A 37, L275-L280 (2004).

[37] M. R. Evans, S. N. Majumdar, and R. K. P. Zia, J. Stat. Phys. 123, 357-390 (2006).

[38] S. Katz, J. L. Lebowitz, and H. Spohn, J. Stat. Phys. 34, 497 (1984).

[39] T. Antal and G. M. Schütz, Phys. Rev. E 62, 83 (2000). 